\title{
Rastreo de cáncer de próstata: continúa la intertidumbre
}

\author{
Prostate cáncer screening: uncertainity continues
}

Djulbegovic M y col.BMJ 2010; 341:c4543 doi: 10.1136/bmj.c4543

\section{Objetivo}

Cuatro años después de la revisión sistemática de la Biblioteca Cochrane ${ }^{1}$ este trabajo analiza la evidencia disponible hasta julio de 2010 acerca de los riesgos y los beneficios de la estrategia: "rastreo de cáncer de próstata $(\mathrm{CP})$ con antígeno prostático específico (en inglés PSA) con o sin tacto rectal", comparada con la estrategia "no rastreo".

Fuentes de datos, selección de estudios y extracción de los datos

Búsqueda electrónica sin restricciones de idioma y tipo de publicación en Medline, Embase, The Cochrane Registry of Controlled Trials (CENTRAL) y en los manuales de resúmenes de las Asociaciones Norteamericana y Europea de Urología y la Sociedad Norteamericana de Oncología Clínica. Fueron seleccionados los estudios aleatorizados y controlados que hubieran comparado el rastreo de CP en hombres asintomáticos con el "no rastreo", excluyendo a los que hubieran incluido pacientes con diagnóstico previo de CP. La estimación de la calidad de los artículos se basó en el Método Cochrane para estimación de sesgos y el Método GRADE y fue realizada por dos revisores independientes, con un tercero para resolver los casos discutidos. Fueron considerados los siguientes resultados: mortalidad por todas las causas y por cáncer de próstata, diagnóstico de cáncer de próstata y estadio al momento del diagnóstico, tasa de falsos positivos y falsos negativos, efectos adversos por el rastreo, calidad de vida, relación costo-beneficio.

\section{Resultados principales}

Fueron incluidos cuatro estudios publicados desde 2009, que sumados a los dos estudios previos que habían integrado la revisión Cochrane en 2006 totalizaron un total de seis estudios con 387.286 participantes: los ensayos 1) Quebec; 2) Norrkoping; 3) Estudio Europeo Aleatorizado de Rastreo de Cáncer de Próstata (ERSPC); 4) el Ensayo Americano de Rastreo de Cáncer de Próstata, Pulmón, Colon y Ovario (PLCO); 5) la rama francesa del ERSPC; y 6) el Ensayo Gothenburg. Sus resultados se resumen en la tabla 1.

Tabla 1: resumen de los principales hallazgos de los seis ensayos clínicos sobre la efectividad del rastreo del cáncer prostático identificados en esta revisión sistemática.

\begin{tabular}{|c|c|c|c|c|c|c|}
\hline \multirow{2}{*}{\multicolumn{2}{|c|}{ Resultados }} & \multicolumn{2}{|c|}{$\begin{array}{l}\text { Incidencia acumulada por } \\
\text { cada mil pacientes* (rango de } \\
\text { seguimiento: } 4 \text { a } 15 \text { años). }\end{array}$} & \multirow[t]{2}{*}{$\begin{array}{l}\text { Riesgo relativo } \\
\text { (IC 95\%) }\end{array}$} & \multirow[t]{2}{*}{$\begin{array}{l}\text { Número de } \\
\text { participantes }\end{array}$} & \multirow[t]{2}{*}{$\begin{array}{l}\text { Calidad de la } \\
\text { evidencia } \\
\text { (GRADE) }\end{array}$} \\
\hline & & Control & Rastreo & & & \\
\hline \multirow[t]{2}{*}{ Mortalidad } & Por todas las causas & 200 & 198 (194 a 202) & $0,99(0,97$ a 1,01$)$ & 256.019 & Moderada \\
\hline & Por cáncer prostático & 8 & $7(6$ a 9$)$ & $0,88(0,71$ a 1,09$)$ & 302.500 & Moderada \\
\hline \multicolumn{2}{|c|}{ Diagnóstico de cáncer de próstata } & 44 & 64 (53 a 78) & $1,46(1,21$ a 1,77$)$ & 340.800 & Baja \\
\hline \multirow{3}{*}{$\begin{array}{l}\text { Estadio al momento } \\
\text { del diagnóstico }\end{array}$} & Estadío I & 11 & 21 (13 a 34) & $1,95(1,22$ a 3,13$)$ & 332.743 & Baja \\
\hline & Estadío II & 23 & 32 (23 a 45) & $1,39(0,99$ a 1,95$)$ & 332.743 & Muy baja \\
\hline & Estadíos III y IV & 5 & 5 (4 a 5) & $0,94(0,85$ a 1,04$)$ & 332.743 & Moderada \\
\hline
\end{tabular}

\section{Conclusiones}

Existe evidencia de moderada calidad para afirmar que no existe un impacto significativo en la mortalidad por todas las causas o por CP atribuible a la estrategia de rastreo; y evidencia de baja calidad que ha documentado un aumento del diagnóstico de CP en los subgrupos sometidos a rastreo, a expensas del diagnóstico en estadío I, lo que no se traduciría en un aumento de la supervivencia global y/o por CP

Palabras claves: rastreo, detección temprana de cáncer y neoplasias de próstata. Keywords: prostate neoplasm, prostate cáncer, screening, early detection.

Fuentes de financiamiento: Departamento de Urología de la Universidad de Florida y Sociedad Americana de Geriatría. No hubo aportes ni relación financiera con ninguna institución que hubiera podido tener interés en el trabajo en los tres años previos a la realización del mismo.

\section{Comentario}

Si bien la incidencia de cáncer de próstata lo convierte en la segunda causa de muerte por cáncer en hombres mayores de 50 años $^{2}$, todavía no se pudo comprobar que el tratamiento precoz en estadíos tempranos de la enfermedad produzca una reducción en su morbilidad y/o mortalidad que justifique los efectos adversos y costos que trae aparejado su diagnóstico temprano ${ }^{3}$.

Dados los resultados de esta revisión sistemática, podemos seguir afirmando que el afán por rastrear una enfermedad que en muchos casos tiene un curso benigno, no haría más que enfermar a pacientes hasta entonces sanos, corriendo el riesgo de someterlos a sobretratamientos, efectos adversos y eventuales daños en su calidad de vida, que todavía no han podido cuantificarse. Por otro lado, no contamos con un método que nos permita diferenciar a aquellos pacientes con CP con progresión benigna de aquellos con una evolución maligna -éste subgrupo sería el potencial beneficiario de las estrategias de rastreo- lo que representa todavía un gran desafío para la investigación.

\section{Conclusión de la comentadora}

Los trabajos disponibles actualmente son escasos, con seguimientos cortos (entre 4 y 15 años) y poseen serias limitaciones metodológicas, por lo que la pregunta acerca de la recomendación del rastreo de cáncer de próstata sigue sin poder responderse. Los resultados de esta revisión sistemática nos hablan de que la evidencia todavía es insuficiente como para respaldar la implementación de esta práctica preventiva.

Cecilia Drimer [ Servicio de Medicina Familiar y Comunitaria de Hospital Italiano de Buenos Aires. cecilia.drimer@ hospitalitaliano.org.ar ]

Drimer C. Rastreo de cáncer de próstata: continúa la intertidumbre. Evid Act Pract Ambul. 13(4).125. Oct-Dic 2010. Comentado de: Djulbegovic M y col. Screening for prostate cancer: systematic review and metaanalysis of randomised controlled trials. BMJ 2010 ; $341: \mathrm{c} 4543$ doi: 10.1136/bmj.c4543. PMID: 20843937.

\section{Referencias}

1. llic D y col. Screening for prostate cancer. Cochrane Database Syst Rev2006;3: CD004720

2. Ministerio de Salud. Dirección de Estadística e Información de Salud. ${ }^{3}$ Ciapponi A.

3. Ciapponi A. El rastreo ("screening") de cáncer de próstata y su eterno dilema: ¿Beneficia o Perjudica? Evid. Act. Pract. Ambul. 12(2). 42-46. Abr-Jun. 2009. 